\title{
DURATION DISTRIBUTION OF THE CONJUNCTION OF TWO INDEPENDENT $F$ PROCESSES
}

\author{
M. T. ALODAT, $* * *$ \\ M. AL-RAWWASH ${ }^{* * * *}$ AND \\ M. A. JEBRINI, ${ }^{* * * * *}$ Yarmouk University
}

\begin{abstract}
In this paper we obtain an approximation for the duration distribution of the excursion set generated by the minimum of two independent $F$ random processes above a high threshold $u$. Moreover, we obtain a closed-form approximation for the mean duration of the conjunction of these two $F$ processes. As an illustration, we conduct a simulation study to compare the performances of the approximated distribution and the exact distribution.
\end{abstract}

Keywords: Conjunction; downcrossing; duration distribution; excursion set; $F$ process; Gaussian process; upcrossing

2000 Mathematics Subject Classification: Primary 60G10

Secondary $60 \mathrm{G} 15$

\section{Introduction}

Random processes such as Gaussian and $F$ processes are widely used in modeling many random responses in various areas of applications, such as engineering. This paper is motivated by a problem in communication engineering where $F$ processes are considered to be flexible models for the load of communication systems. An extreme value of the communication load of a system indicates that the system is unavailable. As a result, the probability that the load of a system exceeds a given threshold is considered to be one of the main communication quality measures. Another significant measure for system quality is the time of unavailability, which is defined as the period of time (duration) that the load spends above a given threshold $u$ after an upcrossing at $u$ (see Figure 1). Leadbetter et al. (1983) studied the problem that involves one Gaussian process and discussed the duration distribution of such a process. To elaborate more on this idea, we assume that $X_{1}(t)$ and $X_{2}(t)$ are the loads of two independent communication systems. We define the duration of unavailability of both systems as the duration of the process $W(t)=\min \left(X_{1}(t), X_{2}(t)\right)$. The problem of deriving an approximation to the duration distribution of $W(t)$ above the threshold $u$ when both processes are independent $F$ processes is an interesting problem that will be discussed in the sequel. Similar ideas and applications can be found in medical, industrial, and other areas of research.

To this end, we assume that $X(t), t \in[0, A]$, is a stationary and differentiable random process with first derivative $\dot{X}(t)$. Accordingly, $X(t)$ will have an upcrossing of $u$ at $t_{0} \in[0, A]$ if $X\left(t_{0}\right)=u$ and $\dot{X}\left(t_{0}\right)>0$. Similarly, $X(t)$ will have a downcrossing of $u$ at $t_{0} \in[0, A]$ if $X\left(t_{0}\right)=u$ and $\dot{X}\left(t_{0}\right)<0$.

Received 5 February 2009; revision received 29 December 2009.

* Postal address: Department of Statistics, Yarmouk University, Irbid, Jordan.

** Email address: malodat@yu.edu.jo

*** Email address: rawwash@yu.edu.jo

**** Email address: mjebrini@ hotmail.com 


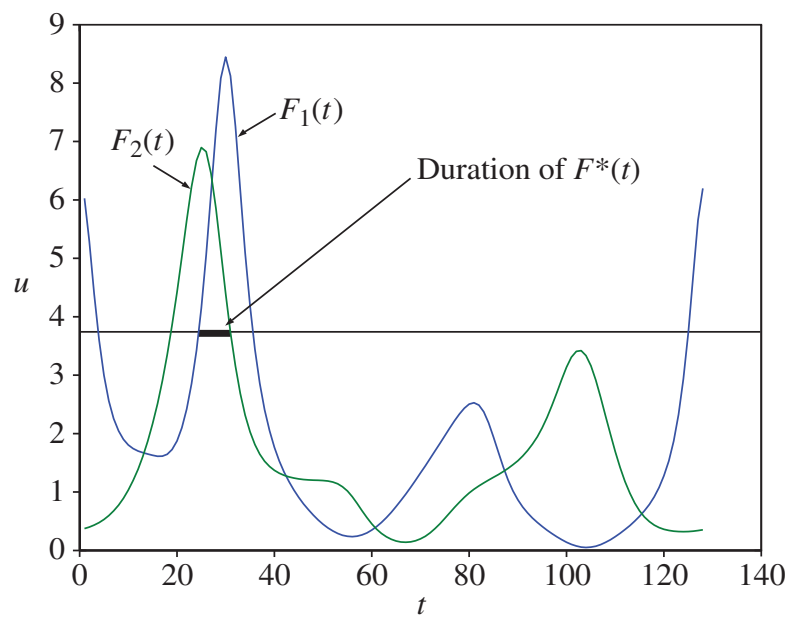

FIGURE 1: The duration of the conjunction of two random processes.

The length of the interval between an upcrossing and the subsequent downcrossing of a level $u$ is called the duration of the excursion of $X(t)$. Moreover, the conjunction of the two random processes $X_{1}(t)$ and $X_{2}(t), t \in[0, A]$, is defined by $X^{*}(t)=\min \left(X_{1}(t), X_{2}(t)\right)$, which happens to be another random process. Many authors have discussed the theory of random processes and the conjunction of two or more Gaussian processes (see Worsley and Friston (2000) and Alodat (2004)). In Figure 1 we clearly show that the excursion set of the conjunction above the threshold $u$ is the intersection of the excursion sets of the processes $X_{1}(t)$ and $X_{2}(t)$ above $u$. In this paper we obtain an approximation to the duration distribution of the excursion of $X^{*}(t)$ when $X_{1}(t)$ and $X_{2}(t)$ are two independent $F$ random processes. The paper is structured as follows. In Section 2 we use Worsley's (1994) definition of the $F$ random process and present some of the main results of Cao (1999) in order to obtain an approximation of the duration distribution of the process. In Section 3 we derive approximations to the durations of two $F$ processes, $F_{1}(t)$ and $F_{2}(t)$; moreover, we obtain the formula that gives the duration of the excursion set of $F^{*}(t)=\min \left(F_{1}(t), F_{2}(t)\right)$ above $u$. In Section 4 we obtain a closed-form approximation for the mean value of the duration of $F^{*}(t)$. In Section 5 we use a simulation to check the validity of our work by comparing the empirical distribution functions with our approximation.

\section{Approximating the duration of $F(t)$}

Suppose that $X_{1}(t), X_{2}(t), \ldots, X_{n}(t)$ and $Y_{1}(t), Y_{2}(t), \ldots, Y_{m}(t)$ are two independent sets of independent, stationary, and real-valued Gaussian random processes with mean 0 and variance 1 . We further assume that the Gaussian random processes used to define $F(t)$ are twice differentiable such that $\lambda=\operatorname{var}\left(\dot{X}_{i}(t)\right)=\operatorname{var}\left(\dot{Y}_{j}(t)\right), i=1,2, \ldots, n$ and $j=1,2, \ldots, m$. We use $\dot{X}(t)$ and $\ddot{X}(t)$ to denote the first and second derivatives of $X(t)$, respectively. Also, we assume that $X_{1}(t), X_{2}(t), \ldots, X_{n}(t)$ and $Y_{1}(t), Y_{2}(t), \ldots, Y_{m}(t)$ are ergodic and satisfy the condition that

$$
\mathrm{E}|\ddot{X}(t)-\ddot{X}(0)| \leq c|t|^{2}
$$


for some $c>0$ and $t$ in some neighborhood of 0. Furthermore, Worsley (1994) defined the $F$ random process as

$$
F(t)=\frac{\sum_{i=1}^{n} X_{i}^{2}(t) / n}{\sum_{j=1}^{m} Y_{j}^{2}(t) / m} .
$$

For a large threshold $u$, the excursion set of a smooth and stationary $F$ random process is a union of disjoint intervals or clusters (see Cao (1999)). Hence, each duration equals the length of one of these intervals. On the other hand, Worsley (1994) gave the following stochastic representation of $\dot{F}(t)$ :

$$
\dot{F}(t)=2 \sqrt{\frac{m}{n}} F^{1 / 2}(t)\left(1+\frac{n}{m} F(t)\right) W^{1 / 2}(t) Z(t),
$$

where $F(t)$ is a Fisher random variable with $n$ and $m$ degrees of freedom, $W(t) \sim \chi_{m+n}^{2}$ and $Z(t) \sim N(0, \lambda)$ such that $F(t), W(t)$, and $Z(t)$ are all independent. According to the result given in (2) and assuming that $G(t)=(n / m) F(t)$, we easily see that

$$
\dot{F}(t)=\frac{m}{n} \dot{G}(t)=\frac{m}{n}\left[2 G^{1 / 2}(t)(1+G(t)) W^{-1 / 2}(t) Z(t)\right] .
$$

For convenience and since $F(t)$ is stationary, we drop the argument $t$ in the rest of the paper. Note that the variance of the random variable $\dot{F}=\dot{F}(t)$ is obtained as follows:

$$
\begin{aligned}
\operatorname{var}(\dot{F}) & =\mathrm{E}\left(\dot{F}^{2}\right)-(\mathrm{E}(\dot{F}))^{2} \\
& =\mathrm{E}\left(4 \frac{m^{2}}{n^{2}} G(1+G)^{2} W^{-1} Z^{2}\right)-\left[\mathrm{E}\left(2 \frac{m}{n} G^{1 / 2}(1+G) W^{-1 / 2} Z\right)\right]^{2} \\
& =4 \frac{m^{2}}{n^{2}} \mathrm{E}\left(G(1+G)^{2}\right) \mathrm{E}\left(W^{-1}\right) \mathrm{E}\left(Z^{2}\right) .
\end{aligned}
$$

Since $G, W$ and $Z$ are independent, it is easy to see that $\mathrm{E}\left(Z^{2}\right)=\lambda$ and, consequently, we have

$$
\mathrm{E}\left(W^{-1}\right)=\frac{\Gamma((m+n) / 2-1)}{2 \Gamma((m+n) / 2)} .
$$

On the other hand, we may write $\operatorname{var}(\dot{F})$ as

$$
\begin{aligned}
\operatorname{var}(\dot{F}) & =4 \frac{m^{2}}{n^{2}} \lambda \mathrm{E}\left(G(1+G)^{2}\right) \frac{\Gamma((m+n) / 2-1)}{2 \Gamma((m+n) / 2)} \\
& =2 \frac{m^{2}}{n^{2}} \lambda \mathrm{E}\left(\frac{n}{m} F_{n, m}\left(1+\frac{n}{m} F_{n, m}\right)^{2}\right)\left(\frac{\Gamma((m+n) / 2-1)}{\Gamma((m+n) / 2)}\right) \\
& =2 \frac{m}{n} \lambda \mathrm{E}\left(F_{n, m}\left(1+\frac{n}{m} F_{n, m}\right)^{2}\right)\left(\frac{\Gamma((m+n) / 2-1)}{\Gamma((m+n) / 2)}\right) .
\end{aligned}
$$

The expectation in (3) requires some numerical computations in order to be evaluated. Cao (1999) introduced the following two theorems concerning the $F$ random process.

Theorem 1. Let $F(t)$ be an $F$ process with a local maximum at $t=0$ and height $F=F(0)$ exceeding $u$. Then, for a given $0<y<1$,

$$
\lim _{u \rightarrow \infty} \mathrm{P}\left(F>(1-y)^{-1} u \mid F>u, \dot{F}=0, \ddot{F}<0\right)=(1-y)^{(m-1) / 2} .
$$


Theorem 2. Conditional on $F=F(0)$, with probability approaching 1 as $u \rightarrow \infty, F(t)$ has the following representation over the cluster of the excursion set containing 0 :

$$
F_{u}(t)=\frac{m A F}{m A+n B t^{2} F}+o(u),
$$

where $F, A$, and $B$ are all independent such that $A \sim \chi_{m+n-1}^{2}$ and $B / \lambda \sim \chi_{m+1}^{2}$.

Using Theorem 2, we can approximate the cluster of $F(t)$ that contains 0 as follows:

$$
\begin{aligned}
B_{u} & =\left\{t: F_{u}(t) \geq u\right\}, \\
& \approx\left\{t: \frac{m A F}{m A+n B t^{2} F} \geq u\right\}, \\
& =\left\{t: m A F \geq m A u+n B t^{2} F u\right\}, \\
& =\left\{t: t^{2} \leq \frac{m A F-m A u}{n u B F}\right\}, \\
& =\left\{t:|t| \leq \sqrt{\frac{m A(F-u)}{n u B F}}\right\} .
\end{aligned}
$$

The interval on the right-hand side of (4) approximates the cluster of $F(t)$ that contains 0 , where $Y=(F-u) / F$. Thus, if $R=\sqrt{m A Y / n u B}$ then $B_{u}=[-R, R]$. In the sequel, we address the problem of obtaining the probability density function (PDF) of $R$.

\section{Approximating the duration of $F^{*}(t)$}

To accomplish the mission of approximating the duration of $F^{*}(t)$, we divide our work into two parts. Steps 1-3 describe the details of obtaining the PDF of $R=\sqrt{m A Y / n u B}$, while step 4 reveals how we obtain the duration of the excursion set of $F^{*}(t)$ above $u$.

Step 1: approximating the durations of $F_{1}(t)$ and $F_{2}(t)$. Let $F_{1}(t)$ and $F_{2}(t), t \in[-L, L]$, be two $F$ processes such that $F_{i}(t)$ has a local maximum at $t_{i}, i=1,2$. Since $F_{i}(t)$ is stationary, we may assume that $t_{i}=0$. Then $F_{i}(t)$ has the following representation near $t_{i}$ :

$$
F_{i, u}(t)=\frac{m A_{i} F_{i}}{m A_{i}+n F_{i} B_{i} t^{2}}+o(u) .
$$

As in (4), we approximate the durations by the intervals $\left[-R_{1}, R_{1}\right]$ and $\left[t_{2}-R_{2}, t_{2}+R_{2}\right]$, where

$$
R_{i}=\sqrt{\frac{m A_{i} Y_{i}}{n u B_{i}}} .
$$

The conjunction $F^{*}(t)$ of $F_{1}(t)$ and $F_{2}(t)$ occurs when the two intervals overlap (see Figure 1). Applying the results of Cao (1999) with $N=1, \Lambda=\lambda$, and $\operatorname{det}\left(b_{i}\right)=b_{i}$ gives the joint horizontal window conditional distribution of $A_{i}$ and $B_{i}$ given that $F_{i}(t)$ has a local maximum which exceeds $u$ at $t_{0}=0$. In other words,

$$
\begin{aligned}
& f_{A_{i}, B_{i}}\left(a_{i}, b_{i} \mid F_{i}=u, \dot{F}_{i}=0, \ddot{F}_{i}<0\right) \\
& \propto a_{i}^{[(m+n-N) / 2]-1} \mathrm{e}^{-a_{i} / 2} \operatorname{det}\left(b_{i}\right)^{(m+1-N-1) / 2} \mathrm{e}^{-\operatorname{tr}\left(-\Lambda^{-1} b_{i}\right) / 2},
\end{aligned}
$$


which reduces to

$$
f_{A_{i}, B_{i}}\left(a_{i}, b_{i} \mid F_{i}=u, \dot{F}_{i}=0, \ddot{F}_{i}<0\right)=\alpha a_{i}^{(m+n-3) / 2} \mathrm{e}^{-a_{i} / 2} b_{i}^{(m-1) / 2} \mathrm{e}^{-b_{i} / 2 \lambda},
$$

where $a_{i}>0, b_{i}>0$, and $\alpha$ is a normalizing constant.

Step 2. Relying on Theorem 1, we derive the cumulative distribution function (CDF) and, hence, the PDF of $Y_{i}=\left(F_{i}-u\right) / F_{i}$, which represents the excess height of $F_{i}(t)$ above $u$. Note that, for $0<y<1$, we have

$$
\mathrm{P}\left(Y_{i} \geq y \mid F_{i}>u, \dot{F}_{i}=0, \ddot{F}_{i}<0\right)=\mathrm{P}\left(F_{i} \geq \frac{u}{1-y} \mid F_{i}>u, \dot{F}_{i}=0, \ddot{F}_{i}<0\right) .
$$

This allows us to conclude that

$$
\lim _{u \rightarrow \infty} \mathrm{P}\left(Y_{i} \geq y \mid F_{i}>u, \dot{F}_{i}=0, \ddot{F}_{i}<0\right)=1-(1-y)^{(m-1) / 2},
$$

which means that the $\mathrm{CDF}$ and $\mathrm{PDF}$ of $Y_{i}$ are respectively

$$
\begin{aligned}
F_{Y_{i}}(y) & = \begin{cases}0, & y<0, \\
1-(1-y)^{(m-1) / 2}, & 0 \leq y<1, \\
1, & y \geq 1,\end{cases} \\
\text { and } f_{Y_{i}}(y) & = \begin{cases}\frac{m-1}{2}(1-y)^{(m-3) / 2}, & 0<y<1, \\
0, & \text { otherwise. }\end{cases}
\end{aligned}
$$

Step 3: finding the PDF of $R_{i}$. To achieve our goal, we first note that

$$
\frac{A_{i}}{B_{i}}=\frac{(m+n-1)}{\lambda(m+1)} W_{i}, \quad \text { where } \quad W_{i}=\frac{A_{i} \lambda(m+1)}{B_{i}(m+n-1)} .
$$

Also, it is well known that $B_{i} / \lambda \sim \chi_{m+1}^{2}$ is equivalent to $B_{i} \sim \Gamma((m+1) / 2,2 \lambda)$, which allows us to conclude that $W_{i} \sim F_{(m+n-1, m+1)}$, the Fisher random variable with $m+n-1$ and $m+1$ degrees of freedom. Consequently, we can rewrite $R_{i}$ as

$$
R_{i}=\sqrt{Y_{i} W_{i} \frac{m}{n u} \frac{(m+n-1)}{\lambda(m+1)}}=c \sqrt{Y_{i} W_{i}}, \quad \text { where } \quad c=\sqrt{\frac{m}{n u} \frac{(m+n-1)}{\lambda(m+1)}} .
$$

Secondly, we need to find the joint PDF of $Y_{i}$ and $W_{i}$, where the PDF of $W_{i}$ is given by

$$
f_{W_{i}}(w)=k w^{(m+n-3) / 2}\left(1+\frac{m+n-1}{m+1} w\right)^{-(2 m+n) / 2}, \quad 0 \leq w<\infty,
$$

where

$$
k=\frac{\Gamma((2 m+n) / 2)}{\Gamma((m+n-1) / 2) \Gamma((m+1) / 2)}\left(\frac{m+n-1}{m+1}\right)^{(m+n-1) / 2} .
$$

Moreover, since $Y_{i}$ and $W_{i}$ are independent, their joint PDF can be written as

$$
f(y, w)=k\left(\frac{m-1}{2}\right)(1-y)^{(m-3) / 2} w^{(m+n-3) / 2}\left(1+\frac{m+n-1}{m+1} w\right)^{-(2 m+n) / 2}
$$

for $w>0$ and $0<y<1$, while $f_{Y_{i}, W_{i}}(y, w)=0$ otherwise. 
Now, we introduce a variable transformation from $(y, w)$ to $(q, r)$, where $q=y$ and $r=c \sqrt{y w}$, with Jacobian equal to $|J|=2 r / c^{2} q$. Thus, the joint PDF of $R_{i}$ and $Q_{i}$ appears as

$$
g(q, r)=\tilde{c} 2 r(1-q)^{(m-3) / 2}\left(\frac{r^{2}}{c^{2} q}\right)^{(m+n-3) / 2}\left(1+\frac{m+n-1}{m+1} \frac{r^{2}}{c^{2} q}\right)^{-(2 m+n) / 2},
$$

where $0<q<1, r>0$, and

$$
\tilde{c}=\frac{\Gamma((2 m+n) / 2)}{\Gamma((m+n-1) / 2) \Gamma((m+1) / 2)}\left(\frac{m-1}{2}\right)\left(\frac{m+n-1}{m+1}\right)^{(m+n-1) / 2} .
$$

Hence, the marginal PDF of $R_{i}$ is obtained by integrating $q$ out. In other words,

$$
g_{R_{i}}(r)=\int_{0}^{1} g(q, r) \mathrm{d} q=\tilde{c}_{1} \frac{1}{r^{m+2}} \Psi\left(\frac{m+3}{2}, m+\frac{n}{2}, m+1, \frac{-m}{r^{2} u \lambda}\right),
$$

where $r>0$,

$$
\tilde{c}_{1}=\frac{2 m^{(m-1) / 2} \Gamma((m+3) / 2) \Gamma(m+n / 2)}{(u \lambda)^{(m+1) / 2} \Gamma(m) \Gamma((m+n-1) / 2)}, \quad \Psi(a, b, c, z)=\sum_{i=0}^{\infty} \frac{(a)_{i}(b)_{i}(z)^{i}}{i !(c)_{i}}
$$

and $(a)_{i}=a(a+1)(a+2) \cdots(a+i-1)$.

Step 4: deriving the duration of the excursion set of $F^{*}(t)$ above $u$. Suppose that $H$ denotes the distance between the centers of the two intervals $I_{1}$ and $I_{2}$, where $I_{1}=\left[-R_{1}, R_{1}\right]$ and $I_{2}=\left[t_{2}-R_{2}, t_{2}+R_{2}\right], t_{2} \in[-L, L]$, are given in step 1. Knowing that the excursion sets of stationary processes are well modeled by a Poisson point process (see Aldous (1989)), then $H$ is uniformly distributed on the interval $[-L, L]$. Accordingly, the conjunction $F^{*}(t)$ occurs if and only if the two intervals overlap, i.e. if the event $G=\left\{0 \leq H \leq R_{1}+R_{2}\right\}$ occurs. Therefore, the duration of the excursion set of $F^{*}(t)$ above $u$ is approximated by

$$
S=2 R_{(1)} \mathbf{1}_{\left[0, R_{(2)}-R_{(1)}\right.}(H)+\left(R_{(1)}+R_{(2)}-H\right) \mathbf{1}_{\left[R_{(2)}-R_{(1)}, R_{(1)}+R_{(2)}\right.}(H),
$$

where $R_{(1)}=\min \left\{R_{1}, R_{2}\right\}, R_{(2)}=\max \left\{R_{1}, R_{2}\right\}$, and $\mathbf{1}_{A}(\cdot)$ denotes the indicator function of $A$. Note that the PDF of $H$ given $R_{1}=r_{1}, R_{2}=r_{2}$, and $G$ is

$$
f_{H}\left(h \mid R_{1}=r_{1}, R_{2}=r_{2}, G\right)=\frac{1}{r_{1}+r_{2}} \mathbf{1}_{\left[0, r_{1}+r_{2}\right]}(h),
$$

while the probability of $G$ given that $R_{1}=v_{1}$ and $R_{2}=v_{2}$ is

$$
\mathrm{P}\left(G \mid R_{1}=v_{1}, R_{2}=v_{2}\right)=\frac{v_{1}+v_{2}}{L} .
$$

Bearing in mind that $R_{1}$ and $R_{2}$ are independent and identically distributed, we easily get

$$
\mathrm{P}\left(R_{1} \leq r_{1}, R_{2} \leq r_{2}, G\right)=\int_{0}^{r_{1}} \int_{0}^{r_{2}} \mathrm{P}\left(G \mid R_{1}=v_{1}, R_{2}=v_{2}\right) g_{R_{1}}\left(v_{1}\right) g_{R_{2}}\left(v_{2}\right) \mathrm{d} v_{1} \mathrm{~d} \nu_{2} .
$$


Consequently, we obtain the probability of $G$ as follows:

$$
\begin{aligned}
\mathrm{P}(G) & =\int_{0}^{\infty} \int_{0}^{\infty} \mathrm{P}\left(G \mid R_{1}=v_{1}, R_{2}=v_{2}\right) g\left(v_{1}\right) g\left(v_{2}\right) \mathrm{d} v_{1} \mathrm{~d} v_{2}, \\
& =\int_{0}^{\infty} \int_{0}^{\infty} \frac{v_{1}+v_{2}}{L} g\left(v_{1}\right) g\left(v_{2}\right) \mathrm{d} v_{1} \mathrm{~d} v_{2}, \\
& =\int_{0}^{\infty} \frac{v_{1}}{L} g\left(v_{1}\right) \mathrm{d} v_{1}+\int_{0}^{\infty} \frac{v_{2}}{L} g\left(v_{2}\right) \mathrm{d} v_{2} \\
& =\frac{2}{L} \int_{0}^{\infty} v_{1} g\left(v_{1}\right) \mathrm{d} v_{1} \\
& =\frac{2}{L} \int_{0}^{\infty} v_{1} \tilde{c}_{1} \frac{1}{v_{1}^{m+2}} \Psi\left(\frac{m+3}{2}, m+\frac{n}{2}, m+1, \frac{-m}{v_{1}^{2} u \lambda}\right) \mathrm{d} v_{1},
\end{aligned}
$$

which leads directly to

$$
\mathrm{P}(G)=\frac{\tilde{c}_{1}}{L} \frac{\sqrt{\pi}(u \lambda)^{m / 2} \Gamma(m) \Gamma((m+n) / 2)}{m^{m / 2} \Gamma((m+3) / 2) \Gamma(m+n / 2)} .
$$

So, the joint CDF of $R_{1}$ and $R_{2}$ given $G$ is

$$
K\left(r_{1}, r_{2} \mid G\right)=\frac{\mathrm{P}\left(R_{1} \leq r_{1}, R_{2} \leq r_{2}, G\right)}{\mathrm{P}(G)},
$$

and the joint PDF of $R_{1}$ and $R_{2}$ given $G$ is

$$
k\left(r_{1}, r_{2} \mid G\right)=(\mathrm{P}(G) L)^{-1}\left(r_{1}+r_{2}\right) g\left(r_{1}\right) g\left(r_{2}\right) .
$$

Having the joint PDF $k\left(r_{1}, r_{2} \mid G\right)$ in the palm of our hands, we focus our attention on simulating from this PDF. In order to carry out this proposed idea, we rewrite $k\left(r_{1}, r_{2} \mid G\right)$ as

$$
k\left(r_{1}, r_{2} \mid G\right)=\frac{r_{1} g\left(r_{1}\right) g\left(r_{2}\right)}{\mathrm{P}(G) L}+\frac{r_{2} g\left(r_{1}\right) g\left(r_{2}\right)}{\mathrm{P}(G) L} .
$$

Then, substituting $\mathrm{P}(G)=(2 / L) \int_{0}^{\infty} r_{1} g\left(r_{1}\right) \mathrm{d} r_{1}$ into the joint PDF $k\left(r_{1}, r_{2} \mid G\right)$ and using (6) allows us to write this joint PDF in the form

$$
k\left(r_{1}, r_{2} \mid G\right)=\frac{r_{1} g\left(r_{1}\right)}{2 \int_{0}^{\infty} r_{1} g\left(r_{1}\right) \mathrm{d} r_{1}} g\left(r_{2}\right)+\frac{r_{2} g\left(r_{2}\right)}{2 \int_{0}^{\infty} r_{2} g\left(r_{2}\right) \mathrm{d} r_{2}} g\left(r_{1}\right) .
$$

Finally, we use (5) to write the joint PDF of $R_{1}, R_{2}$, and $H$ given $G$ as

$$
\begin{aligned}
D\left(r_{1}, r_{2}, h \mid G\right) & =f\left(h \mid R_{1}=r_{1}, R_{2}=r_{2}, G\right) k\left(r_{1}, r_{2} \mid G\right) \\
& =\frac{1}{r_{1}+r_{2}} \mathbf{1}(h)_{\left[0, r_{1}+r_{2}\right]} k\left(r_{1}, r_{2} \mid G\right) .
\end{aligned}
$$

\section{Duration mean}

In this section we rely on the notation and results presented in step 4 to obtain the mean value of the duration $S$. To proceed in this direction, we assume that $I_{1}$ and $I_{2}$ are two intervals with lengths $2 l_{1}$ and $2 l_{2}$, respectively, such that $I_{1}$ is fixed. On the other hand, $I_{2}$ moves uniformly 
around $I_{1}$ such that $I_{2}$ meets $I_{1}$. From the classical geometric probability theory, the mean value of the overlap length of $I_{1}$ and $I_{2}$ is

$$
\frac{2 l_{1} l_{2}}{l_{1}+l_{2}} .
$$

In our case, $l_{1}=R_{1}$ and $l_{2}=R_{2}$ are considered to be random variables. To find $\mathrm{E}(S \mid G)$, we first condition on $R_{1}$ and $R_{2}$, and then we take the expectation with respect to $R_{1}$ and $R_{2}$. If $I_{1}=\left[-R_{1}, R_{1}\right]$ and $I_{2}=\left[t_{2}-R_{2}, t_{2}+R_{2}\right]$, as described in Section 3, then we use the iterated expectations to obtain

$$
\begin{aligned}
\mathrm{E}(S \mid G) & =\mathrm{E}_{R_{1}, R_{2}}\left(\mathrm{E}_{H}\left(S \mid R_{1}, R_{2}, G\right)\right) \\
& =\mathrm{E}_{R_{1}, R_{2}}\left(\frac{2 R_{1} R_{2}}{R_{1}+R_{2}} \mid G\right) \\
& =(\mathrm{P}(G) L)^{-1} \int_{0}^{\infty} \int_{0}^{\infty} \frac{2 r_{1} r_{2}}{r_{1}+r_{2}}\left(r_{1}+r_{2}\right) g\left(r_{1}\right) g\left(r_{2}\right) \mathrm{d} r_{1} \mathrm{~d} r_{2} \\
& =2(\mathrm{P}(G) L)^{-1} \int_{0}^{\infty} r_{1} g\left(r_{1}\right) \mathrm{d} r_{1} \int_{0}^{\infty} r_{2} g\left(r_{2}\right) \mathrm{d} r_{2} \\
& =\frac{2}{L \mathrm{P}(G)}\left(\int_{0}^{\infty} r_{1} g\left(r_{1}\right) \mathrm{d} r_{1}\right)^{2} \\
& =\int_{0}^{\infty} r_{1} g\left(r_{1}\right) \mathrm{d} r_{1} \\
& =\int_{0}^{\infty} r_{1} \tilde{c}_{1} \frac{1}{r_{1}^{m+2}} \Psi\left(\frac{m+3}{2}, m+\frac{n}{2}, m+1, \frac{-m}{r_{1}^{2} u \lambda}\right) \mathrm{d} r_{1},
\end{aligned}
$$

which allows us to conclude that

$$
\mathrm{E}(S \mid G)=\sqrt{\frac{\pi}{m u \lambda}} \frac{\Gamma((m+n) / 2)}{\Gamma((m+n-1) / 2)} .
$$

\section{Simulation study}

To check the validity of our approximation, we compare the empirical distribution functions of two large samples, one of which is obtained from the approximation and the other from the exact distribution. Since the exact duration distribution is unknown, we obtain a large sample from it by simulating a Gaussian process. We generate 5000 samples that correspond to different values of the threshold, $u=1,2,3,4,5,6$, and 7 . Finally, we design the following algorithm to simulate from the distribution of $S$.

1. Simulate $\left(R_{1}, R_{2}\right)$ from $k\left(r_{1}, r_{2} \mid G\right)$.

2. Simulate $H$ from $f_{H}\left(h \mid R_{1}=r_{1}, R_{2}=r_{2}, G\right)$.

3. The random vector $\left(R_{1}, R_{2}, H\right)$ is distributed according to $D\left(r_{1}, r_{2}, h \mid G\right)$.

4. Obtain $S=2 R_{(1)} \mathbf{1}_{\left[0, R_{(2)}-R_{(1)}\right]}(H)+\left(R_{(1)}+R_{(2)}-H\right) \mathbf{1}_{\left[R_{(2)}-R_{(1)}, R_{(1)}+R_{(2)}\right.}(H)$.

To conduct our simulation in step one, we use the Metropolis algorithm (see Robert and Casella (1999)). This algorithm focuses on simulating from the density $h(x)$ by following the steps listed below. 
1. Select an initial value $\theta_{0}$.

2. Generate $\zeta$ uniformly in a neighborhood of $\theta_{0}$.

3. The new value of $\theta$ is updated in the following manner:

$$
\theta_{1}= \begin{cases}\zeta & \text { with probability } \rho=\min \{\exp (\Delta h / T), 1\} \\ \theta_{0} & \text { with probability } 1-\rho,\end{cases}
$$

where $\Delta h=h(\zeta)-h\left(\theta_{0}\right)$.

Tables 1-3 show the exact and approximate means of $S$, and Figures 2-7 illustrate the empirical distribution functions of both samples.

TABLE 1: The exact and approximate means when $n=3$ and $m=10$.

\begin{tabular}{ccc}
\hline$u$ & Exact & $\mathrm{E}(S \mid G)$ \\
\hline 1.0 & 1.1147 & 0.5944 \\
2.0 & 0.5169 & 0.4203 \\
3.0 & 0.3600 & 0.3432 \\
4.0 & 0.2910 & 0.2972 \\
5.0 & 0.2522 & 0.2658 \\
6.0 & 0.2225 & 0.2427 \\
7.0 & 0.2061 & 0.2247 \\
\hline
\end{tabular}

TABLE 2: The exact and approximate means when $n=5$ and $m=10$.

\begin{tabular}{ccc}
\hline$u$ & Exact & $\mathrm{E}(S \mid G)$ \\
\hline 1.0 & 1.2055 & 0.5487 \\
2.0 & 0.5799 & 0.3880 \\
3.0 & 0.3952 & 0.3168 \\
4.0 & 0.3138 & 0.2743 \\
5.0 & 0.2582 & 0.2454 \\
6.0 & 0.2327 & 0.2240 \\
7.0 & 0.2100 & 0.2074 \\
\hline
\end{tabular}

TABLE 3: The exact and approximate means when $n=7$ and $m=10$.

\begin{tabular}{ccc}
\hline$u$ & Exact & $\mathrm{E}(S \mid G)$ \\
\hline 1.0 & 0.6077 & 0.6369 \\
2.0 & 0.4134 & 0.4503 \\
3.0 & 0.3677 & 0.3691 \\
4.0 & 0.3308 & 0.3677 \\
5.0 & 0.3043 & 0.2848 \\
6.0 & 0.2763 & 0.2600 \\
7.0 & 0.2550 & 0.2407 \\
\hline
\end{tabular}



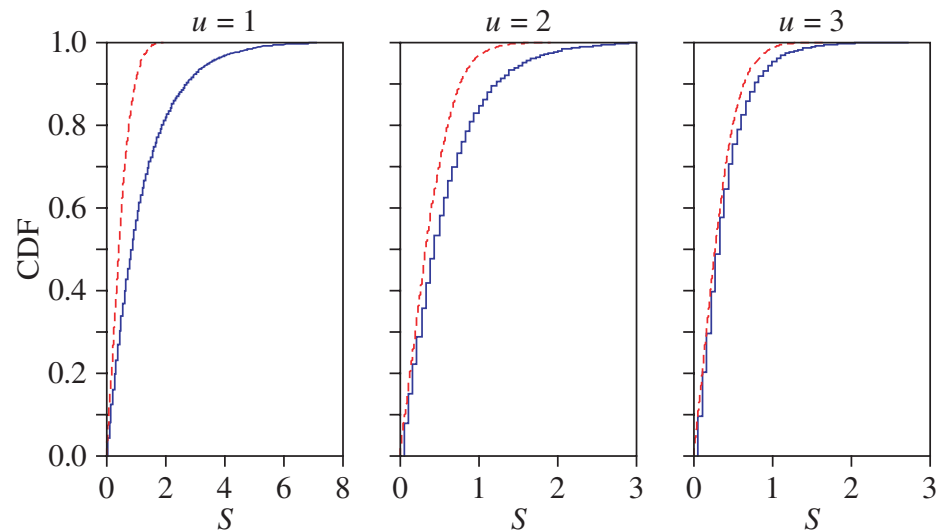

Figure 2: The exact CDF (solid line) and the approximation CDF (dashed line) of $S$ when $u=1,2,3$, $n=3$, and $m=10$.
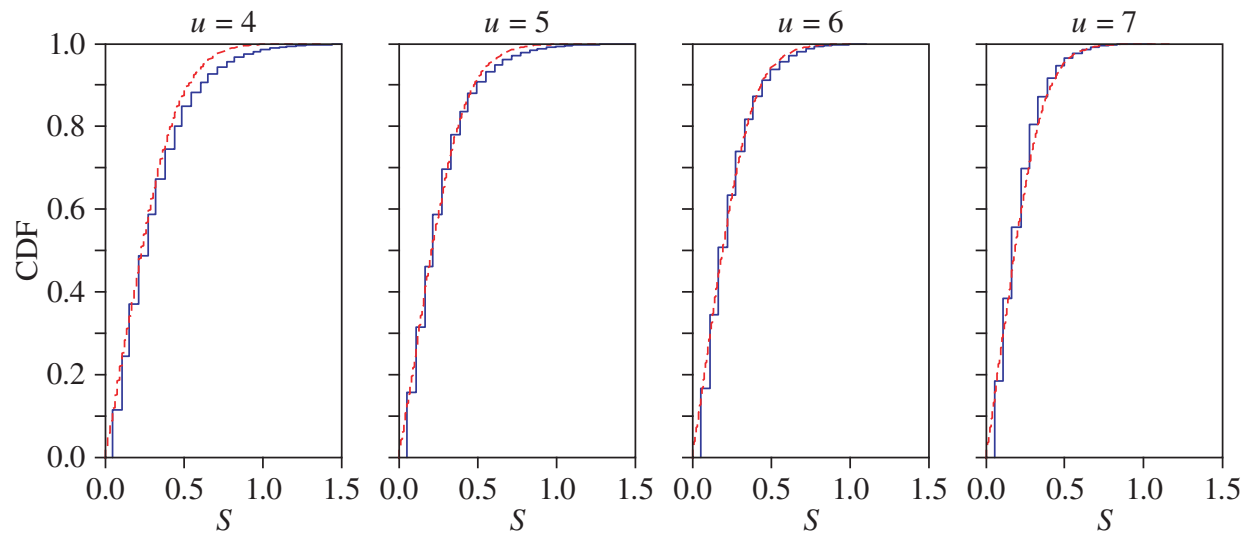

Figure 3: The exact CDF (solid line) and the approximation CDF (dashed line) of $S$ when $u=4,5,6,7$, $n=3$, and $m=10$.
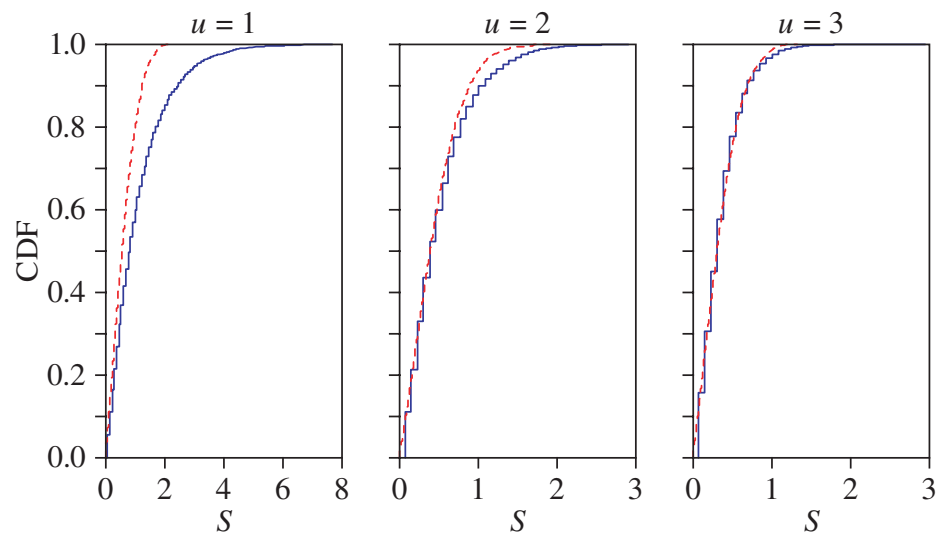

FIGURE 4: The exact CDF (solid line) and the approximation CDF (dashed line) of $S$ when $u=1,2,3$, $n=5$ and $m=10$. 

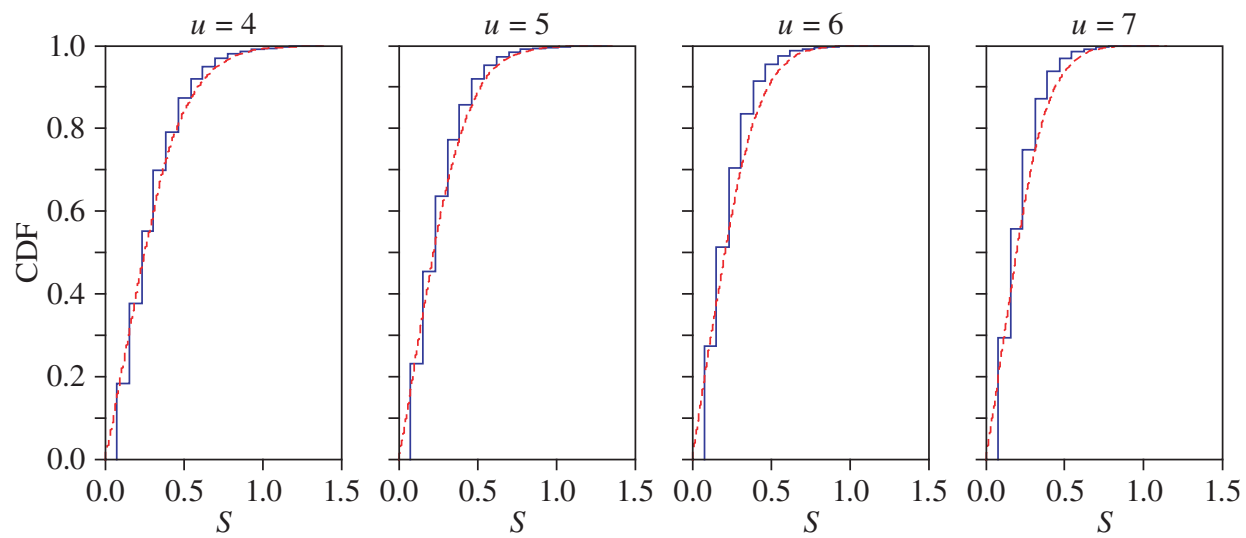

Figure 5: The exact CDF (solid line) and the approximation CDF (dashed line) of $S$ when $u=4,5,6,7$, $n=5$, and $m=10$.


Figure 6: The exact CDF (solid line) and the approximation CDF (dashed line) of $S$ when $u=1,2,3$, $n=7$ and $m=10$.
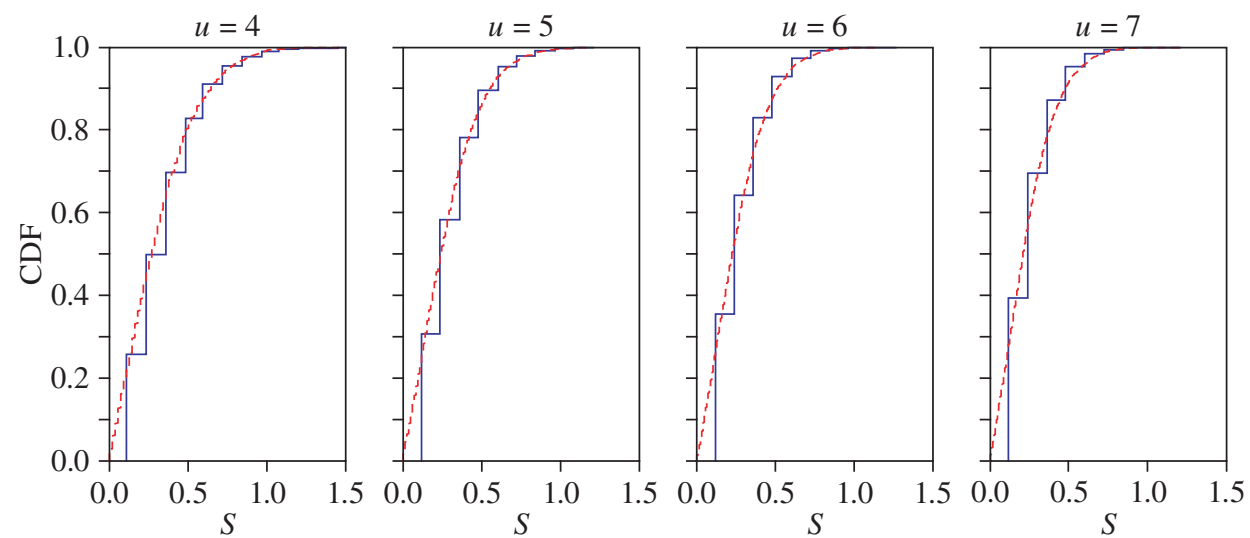

Figure 7: The exact CDF (solid line) and the approximation $\operatorname{CDF}$ (dashed line) of $S$ when $u=4,5,6,7$, $n=7$ and $m=10$. 
The simulation results in Tables 1-3 as well as the distribution functions shown in Figures 2-7 show that the approximation works well for large values of $u$; moreover, we note that our approximation approaches the exact approximation as the values of $u$ and $n$ increase.

\section{References}

Aldous, D. (1989). Probability Approximations via the Poisson Clumping Heuristic. Springer, New York. Alodat, M. T. (2004). Detecting conjunctions using cluster volumes. Doctoral Thesis, McGill University.

Cao, J. (1999). The size of the connected components of excursion sets of $\chi^{2}, t$ and $f$ fields. Adv. Appl. Prob. 31, $579-595$.

Leadbetter, M. R., Lindgren, G. And Rootzén, H. (1983). Extremes and Related Properties of Random Sequences and Processes. Springer, New York.

Robert, C. P. and Casella, G. (1999). Monte Carlo Statistical Methods. Springer, New York.

Worsley, K. J. (1994). Local maxima and the expected Euler characteristic of excursion sets of $\chi^{2}, t$ and $f$ fields. Adv. Appl. Prob. 26, 13-42.

Worsley, K. J. And Friston, K. J. (2000). A test for a conjunction. Statist. Prob. Lett. 47, 135-140. 